\title{
Study on polarization generator based on electro-optic modulation
}

\author{
Dongmei $\mathrm{Hu}^{1,2, a}$, Lu Song ${ }^{1, b}$ and Guocheng Niu${ }^{2, \mathrm{c}}$ \\ ${ }^{1}$ College of Electronic Information Engineering, Changchun University Science and Technology, \\ Changchun, China \\ 2 College of Electrical and Information Engineering, Beihua University, Jilin, China \\ aemail: haitianme@163.com, bemail:usong@126.com, cemail:niuguochengjilin@163.com
}

\begin{abstract}
Keywords: Modulation of Polarization Direction; State of Polarization(SOP); Liquid Crystal Variable Retarder(LCVR); Stokes Vectors
\end{abstract}

\begin{abstract}
Polarization state generator is an indispensable part of polarization imaging system, produces the state of polarization for the calibration or test . Based on the analysis of the existing scheme, a novel Polarization State Generator is developed, which is constituted of a linear polarizer $\mathrm{P}$ and two liquid crystal phase variable retarders, denoted as LCVR1 and LCVR2. The transmission axes of P and LCVR2 are oriented horizontally, while the fast axes of LCVR2 is oriented. the state of polarization of the incident light is modulated through adjusting the control voltage of LCVRS. the experimental results show that the system not only is feasible ,but also has high sensibility. A good agreement is observed between theoretical and experimental results.
\end{abstract}

\section{Introduction}

Polarization generator is an indispensable part of polarization imaging system, in order to measure the instrument polarization sensitivity and monitor instrument performance requirements change, has the extremely high sensitivity. Laser is a common light source in polarization calibration, therefore, the polarizer , plate or other optical devices are placed in the laser beam path , thus can obtain the required for various polarization state laser beam [1 ].

In this paper, Based on the Stokes vector and Muller matrix theory, the traditional measurement technique, rotating wave plate driven by a motor, is replaced with Liquid crystal variable retarders (LCVRS), the phase retardation is controlled by the applied voltage, the polarization state of incident light is modulated accurately, eliminating the mechanical error, the polarization of laser generated is more accurate.

\section{Theory}

The Stokes Vectors of the light can be used to characterize the polarization properties of the light beam. The elements with the strength of the dimension, are all real Numbers, and can be easily measured and calculated, in the practical process of polarization detection, stokes vectors has become an important method to determine the polarization of the laser beam.

In the Mueller calculus, the Stokes vector is used to described the polarization state of a light beam(where $\mathrm{T}$ denotes transposition). where is the total light intensity, the difference between the intensity of the horizontal (X) polarized component and that of the vertical (Y) linearly polarized component, the difference between the intensity of the linearly polarized component oriented at an angle of and that of the linearly polarized component oriented at an angle of , and the difference between the intensity of the left-circularly polarized component and that of the right-circularly polarized component[2-4].

The Stokes vector of the output beam can be written as:

$S_{\text {out }}=M \times S_{\text {in }}$

where $S_{i n}$ is the Stokes vector of the input beam and M is Mueller matrices [5] of n optical elements respectively given by $\mathrm{M}=M_{n} M_{n-1} \ldots M_{2} M_{1}$ 
Degree of Polarization(DOP),Linear Polarization(DOLP), Circular Polarization (DOCP), azimuth $(\theta$ )and ellipticity $(\varepsilon)$ can be obtained as:

$$
\begin{aligned}
& D O P=\left(\sqrt{S_{1}^{2}+S_{2}^{2}+S_{3}^{2}} / S_{0}\right) \times 100 \% \\
& D O L P=\left(\sqrt{S_{1}^{2}+S_{2}^{2}} / S_{0}\right) \times 100 \% \\
& \theta=\frac{1}{2} \arctan \left(S_{2} / S_{1}\right) \quad[-\pi / 2, \pi / 2] \\
& \varepsilon=\frac{1}{2} \arcsin \left(S_{3} / \sqrt{S_{1}^{2}+S_{2}^{2}+S_{3}^{2}}\right) \quad[-\pi / 4, \pi / 4]
\end{aligned}
$$

\section{Institutions Optimization Design}

The commonly used PSG is constituted of a linear polarizer $\mathrm{P}$ and a quarter-wave plate . Line polarized light can be produced by adjusting P. To produce a left-handed and a right-handed circular polarized light, the transmission axes of $\mathrm{P}$ is oriented horizontally , while the fast axes of the quarter-wave plate is oriented $\pm \pi / 4$. The disadvantages is big volume, slow response, great mechanical error and so on.The rotating-retarder configuration can be replaced by one liquid crystal variable retarder[3] for determination of four complete Stokes vectors. The configuration greatly improves the response speed, improves accuracy, decreases the influence of mechanical rotating at the same time, but not completely eliminated.

So the scheme based on dual LCVRS is presented. The system is constituted of a linear polarizer $\mathrm{P}$ and two liquid crystal phase variable retarders, denoted as LCVR1 and LCVR2, the structure diagram is shown in figure 1.

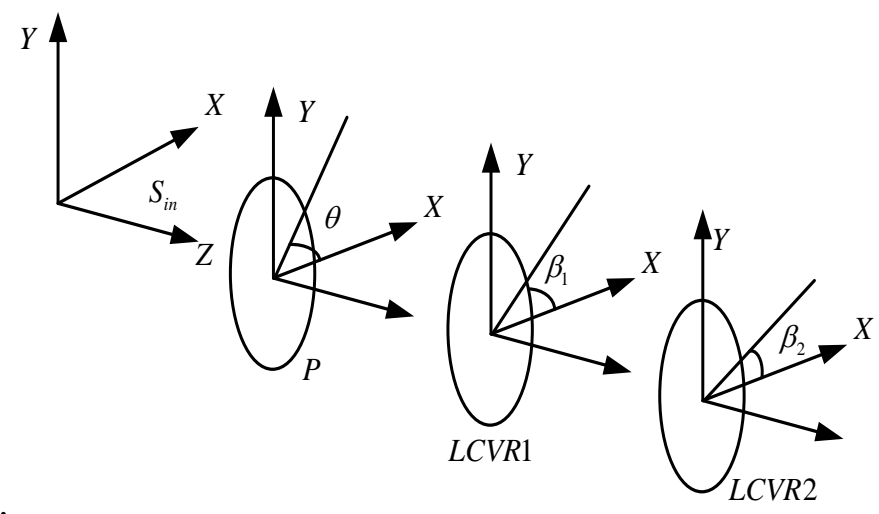

Fig.1. Coordinate system structure diagram of the polarization system based on dual LCVRS The Stokes vector of the output beam can be written as:

$$
S_{\text {out }}=M_{2}\left(\beta_{2}, \delta_{2}\right) M_{1}\left(\beta_{1}, \delta_{1}\right) M_{P}(\theta) S_{\text {in }}
$$

where $S_{i n}$ is the Stokes vector of the input beam, and given by $S_{i n}=\left[\begin{array}{llll}S_{0}^{\prime} & S_{1}^{\prime} & S_{2}^{\prime} & S_{3}^{\prime}\end{array}\right]^{T}$, $M_{P}(\theta), M_{i}\left(\beta_{i}, \delta_{i}\right) \quad(i=1,2)$ are Mueller matrices[5] of the polarizer and retarders respectively , $\delta$ represents the phase retardation, $\theta$ gives the angle between the fast axis of the polarizer and the horizontal axis, $\beta$ gives the angle between the fast axis of the retarders and the horizontal axis.

When the azimuth of the polarizer and retarders respectively is given by $\theta=0^{\circ} 、 \beta_{1}=45^{\circ}$ 、 $\beta_{2}=0^{\circ}$,

$M_{1}\left(\beta_{1}, \delta_{1}\right)=\left[\begin{array}{cccc}1 & 0 & 0 & 0 \\ 0 & \cos \delta_{1} & 0 & -\sin \delta_{1} \\ 0 & 0 & 1 & 0 \\ 0 & \sin \delta_{1} & 0 & \cos \delta_{1}\end{array}\right]$ and $M_{2}\left(\beta_{2}, \delta_{2}\right)=\left[\begin{array}{cccc}1 & 0 & 0 & 0 \\ 0 & 1 & 0 & 0 \\ 0 & 0 & \cos \delta_{2} & \sin \delta_{2} \\ 0 & 0 & -\sin \delta_{2} & \cos \delta_{2}\end{array}\right]$ 
After normalized , $S_{\text {out }}=\left[\begin{array}{llll}1 & \cos \delta_{1} & \sin \delta_{1} \sin \delta_{2} & \sin \delta_{1} \cos \delta_{2}\end{array}\right]^{T}$

The theoretical study shows, the polarized light source is required through adjusting the phase retardation of LCVRS. then, LCVRS is the electrically tunable phase delayer, through changing the voltage across the LCVRS, phase delay of different incident wavelength can be achieved, so the polarization generator accuracy is determined by the photoelectric characteristics of LCVRS.

\section{Calibration of the liquid crystal variable retarder.}

In order to realize the precise modulation of the laser polarization state, the calibration must be performed on the LCVRS, namely the problem with LCVRS is the non-linear relation between phase retardance and applied voltage[6-7], the principle diagram for LCVRS phase delay characteristic measuring system is shown in figure 2.

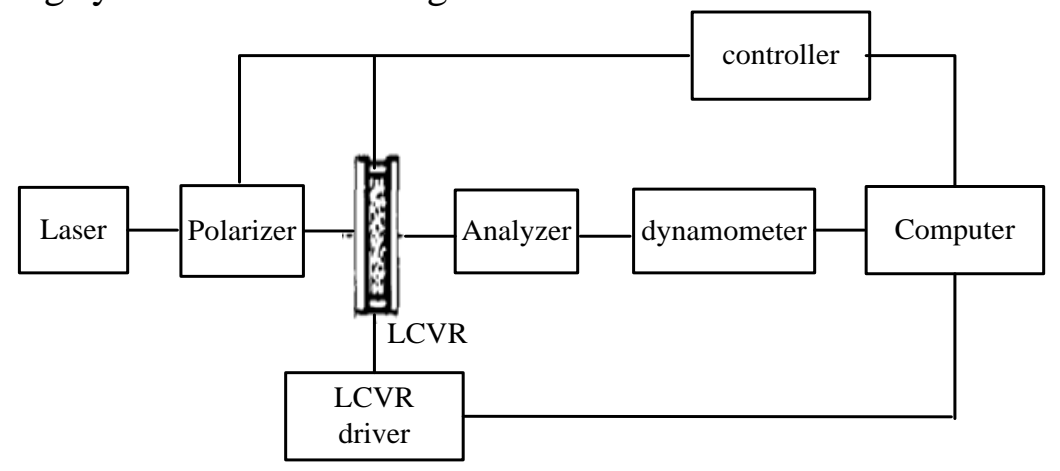

Figure.2. the principle diagram for LCVR phase delay characteristic measuring system.

First, the transmission axes of the polarizer and analyzer are oriented horizontally ,while the fast axes of LCVR is oriented $45^{\circ}$. Second, adjusting the laser, so that the output wavelength is the wavelength to be measured, makeing the laser vertically into the polarizer by means of optical path adjustment. Finally, by using the power meter to measure the maximum intensity $I_{\max }$, when $\delta=0^{\circ}$, the value of output light intensity is strongest. According to Stokes vector and matrix theory, the equation between the output light intensity and phase retardation is $I=\mathrm{a}(1+\cos \delta)$, while $a=0.5 I_{\max }$, thus, the phase delay characteristic curve is simulated by MATLAB.

The experiment selected $438 \mathrm{~nm}, 532 \mathrm{~nm}, 676 \mathrm{~nm}$ three bands for polarization test, in order to ensure the accuracy of LCVRS polarization modulation, the incident angle of the incident light is less than $5^{\circ}$,the simulation curve is shown in figure 3.

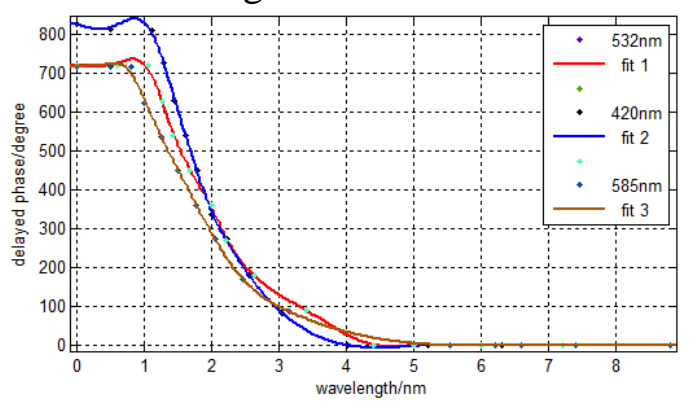

Figure.3. the phase delay characteristic curve of different wavelength

The above curves show that delayed Phase characteristic of LCVRS for the incident light with different wavelengths is different, when LCVRS applied voltage exceeds a threshold voltage (about $1 \mathrm{~V}$ ), the amount of delay begins to decrease with the increase of voltage; in the range of $1 \mathrm{~V}-2.6 \mathrm{~V}$, the characteristics is linear and easy to control, try to make the liquid crystal to work in this range.

\section{Data Reduction and Analysis}

In this paper, aiming at the wavelength of 532nm laser ,after calibration of LCVR , obtained corresponding to the driving voltage of $0 、 \lambda / 4 、 \lambda / 2$ 和 $3 \lambda / 4$, the driving voltage control of two 
LCVRS by computer. test data of some special polarization is shown in table 1. Table 1 test data of some special polarization

\begin{tabular}{|c|c|c|c|c|c|c|c|c|c|c|}
\hline \multirow{2}{*}{\multicolumn{2}{|c|}{ Light }} & \multicolumn{4}{|c|}{ Stokes } & \multicolumn{2}{|l|}{ SOP } & \multicolumn{3}{|l|}{ DOP } \\
\hline & & $S_{0}$ & $S_{1}$ & $S_{2}$ & $S_{3}$ & $\varepsilon$ & $\theta$ & $D O P(\%)$ & $\operatorname{DOLP}(\%)$ & $D O C P(\%)$ \\
\hline \multirow{4}{*}{$\begin{array}{c}\text { line polarize } \\
\text { light }\end{array}$} & $\mathrm{O}^{\circ}$ & 1 & 1 & 0.008 & -0.009 & 0.036 & 0.920 & 100 & 100 & 0.9 \\
\hline & $90^{\circ}$ & 1 & 0 & 1 & 0.001 & 0.025 & 44.989 & 100 & 100 & 0.1 \\
\hline & $45^{\circ}$ & 1 & -1 & -0.019 & 0.001 & 0.053 & 89.610 & 100.018 & 100.018 & 0.1 \\
\hline & $135^{\circ}$ & 1 & 0.001 & -1 & -0.003 & -0.108 & 134.973 & 100 & 100 & 0.3 \\
\hline \multirow{2}{*}{$\begin{array}{c}\text { rotatory } \\
\text { polarization }\end{array}$} & $\mathrm{L}$ & 1 & -0.013 & -0.001 & -1 & -44.645 & 1.0372 & 100 & 1.3 & -100 \\
\hline & $\mathrm{R}$ & 1 & -0.032 & -0.002 & 0.999 & 44.057 & 1.9414 & 99.951 & 3.2 & 99.9 \\
\hline
\end{tabular}

The table shows that the measurement error is about $0.1 \%$, the system accuracy is mainly affected by the random error,In order to overcome the error, we ran it multiple metering and took the average.

\section{Conclusions}

In the present paper, a new system to generate the required polarized light has been developed. Through theoretical derivation, numerical calculation and experiment shows that the polarization converter can realize the full range of output polarization state with lower voltage, and can meet the need of different wavelength laser phase delay, can obtain the accurate, stable and adjustable all kinds of laser polarization. the advantages are small volume, low driving voltage, fast response speed, dynamic continuous adjustable and large adjustable range, easy control, no mechanical adjustment, long life etc. Therefore, laser phase modulation based on dual LCVRS is feasible and has the important research value, provides higher efficiency and accuracy of measurement.

\section{ACKNOWLEDGMENT}

Supported by the national 973 project fund(613225).

\section{Reference}

[1] Gilman S E, Baur T G, Gallagher D J, et al.. Properties of tunable nematic liquid-crystal retarders[J]. SPIE, 1990,1166: 461 471

[2] Clarke D, Grainger J F. Polarized light and optical measurement [M]. Oxford: Pergamon Press, 1971: 56-58

[3] Liao Yanbiao. The polarization of optics [M]. Beijing: Science Press, 2003: 52-56, 243-244.

[4]Marenori Kawamura, Yoshiaki Goto, Susumu Sato. Two-dimensional measurements of cell parameter distributions in reflective liquid crystal displays by using multiple wavelengths Stokes parameters[J]. Journal of Applied Physics, 2004, 95(8): 4371-4375.

[5] Juan M B. Polarimetry using liquid-crystal variable retarders: theory and calibration[J]. Opt A: Pure Appl Opt, 2000, 2(3): 216 222.

[6] Azzam R M A. Arrangement of four photo-detectors for measuring the state of polarization of light [J]. OPTICS LETTERS, 1985, 10(7): 309-311.

[7] YU Hui. Study on dispersion characteristics of LCVR in visible light[J]. Journal of Shanxi University (Nat. Sci. Ed.), 2014, 37(4): 539-544.

[8] ZHANG Ying, ZHAO Huijie, ZHOU Pengwei, et al.. Photoelectric characteristics of liquid crystal variable retarder[J]. Foreign Electronic Measurement Technology, 2009, 28(3): 17-20. 